\title{
Cleidotrapezius: An Unusual Variant Slip from the Sternocleidomastoid
}

\author{
The Editor,
}

Sir,

Abnormal origin, presence of additional bellies and slips and layered arrangement of fibres are common variations of the sternocleidomastoid muscle. Variations are more common in the cleidomastoid portion of the muscle (1). During routine dissection classes in the Department of Anatomy, Kasturba Medical College, Manipal, a rare variation was encountered on the left-side of the neck of a 62-year old male cadaver. A muscle slip about 0.5 $\mathrm{cm}$ thick was arising from the clavicle very close and lateral to the clavicular part of the sternocleidomastoid. This slip further crossed the posterior triangle transversely across the trunks of the brachial plexus and inferior belly of the omohyoid muscle, passing deep to the external jugular vein but superficial to the supraclavicular nerves.

The external jugular vein was passing through a small gap between this additional muscle slip and the clavicle to drain into the subclavian vein. The muscle fasciculus finally flattened out and was blending with the anterior border of the trapezius (Figure) about $3 \mathrm{~cm}$ above the clavicle. The nerve supply, when traced, was provided by the spinal accessory nerve. No other associated variations were observed in the neck region of the same cadaver.

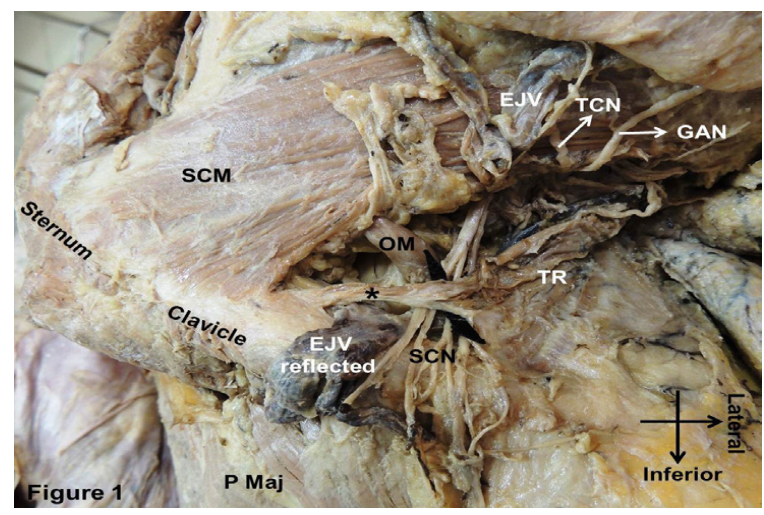

Figure: An unusual variant slip arising from the sternocleidomastoid and blending with the trapezius: A muscle slip $(*)$ arising from the clavicle very close to the clavicular part of the sternocleidomastoid (SCM). This slipfurther crossed the posterior triangle upward and backward, passing deep to the external jugular vein (EJV) but superficial to the supraclavicular nerves $(\mathrm{SCN})$. The muscle fasciculus finally flattened out and was blending with the anterior border of the trapezius (TR). OM: inferior belly of omohyoid, GAN: great auricular nerve, TCN: transverse cutaneous nerve of neck.

Developmentally, sternocleidomastoid muscle (SCM) shares a common origin with the trapezius from the posterior sixth branchial arch. These additional muscle slips indicate abnormal mesodermal splitting (2). Additional slips of SCM although asymptomatic, may sometimes lead to compression of the underlying neurovascular structures. It can even cause severe clinical symptoms like sternomastoid pseudotumours and congenital torticollis, and should be studied in detail for their prompt management (3). In the present case, the muscle slip presented superficial to the supraclavicular nerves and deep to the external jugular vein. Since it covers the important neurovascular structures in the lower part of the neck, it might cause difficulties in surgeries in that region and may also interfere during the use of different invasive techniques. It may even cause significant stenosis of the lesser supraclavicular fossa, imposing complications for anaesthesiologists during the anterior central venous catheterization approach.

However, it can be effectively utilized for muscle flap harvests (4). Earlier, additional slips of SCM were shown to be beneficial as they can be used as a muscle and myocutaneous flap in the treatment of oral cavity reconstruction, facial deficits and as a gator neck flap in neck dissections (5). Thorough knowledge of variations of the sternocleidomastoid muscle is therefore necessary for harvesting muscle flap for reconstructive surgeries, to relieve neurovascular compression symptoms and to avoid possible complications during surgeries and other invasive techniques. 
Keywords: Cleidotrapezius, myocutaneous flaps, neurovascular compression, sternocleidomastoid, supraclavicular nerves, trapezius

SR Kotian, SK Mittal, KMR Bhat

From: Department of Anatomy, Kasturba Medical College, Manipal, Karnataka, India.

Correspondence: Dr KMR Bhat, Department of Anatomy, Kasturba Medical College, Manipal-576104, India. Fax-+91-820-2570061; e-mail:

kumar.mr@manipal.edu

DOI: 10.7727/wimj.2014.186

\section{REFERENCES}

1. Williams PL Gray's Anatomy. 38th, Edinburgh: Churchill Livingstone; 1995: 804-5.

2. Hasan T. Variations of the sternocleidomastoid muscle: a literature review. Internet J Hum Anat 2011:1.

3. Cheng JC, Tang SP, Chen TM. Sternocleidomastoid pseudo tumor and congenital muscular torticollis in infants: a prospective study of 510 cases. J Pediatr 1999; 134: 712-6.

4. Cherian SJ, Nayak S. A rare case of unilateral third head of sternocleido mastoid muscle. Int J Morphol 2008; 26: 99-101.

5. Parell GJ, Cassisi NJ. The skin-sternocleidomastoid muscle composite flap for neck dissection (the gator neck flap). Laryngoscope 2008; 118: 1791-2. 